\title{
Review of Allopregnanolone Agonist Therapy for the Treatment of Depressive Disorders
}

\author{
Autumn Walkery (D) \\ Lauren D Leader $\mathbb{D}^{\prime}$ \\ Emily Cooke (D) $^{2}$ \\ Amy VandenBerg' \\ 'Department of Pharmacy Services, \\ Michigan Medicine, Ann Arbor, MI, USA; \\ ${ }^{2}$ Department of Pharmacy, Barnes-Jewish \\ Hospital, St. Louis, MO, USA
}

Objective: This paper reviews the current literature available for the efficacy and safety of allopregnanolone agonists and discusses considerations for their place in therapy.

Literature Search: A literature search was conducted utilizing PubMed, clinicaltrials.gov, and the manufacturer's website.

Data Synthesis: One phase II trial and two phase III trials evaluating the efficacy and safety of brexanolone were identified. Brexanolone demonstrated efficacy through significantly reduced Hamilton Depression Rating Scale (HAM-D) scores compared to placebo in the treatment of postpartum depression (PPD). Noted adverse effects were somnolence and dizziness, excessive sedation, and loss of consciousness. One published phase II study and the interim results of two phase III trials and one phase II trial on zuranolone were included in this review. Zuranolone, an oral allopregnanolone agonist, is given as a single, 14-day course. A significant reduction in HAM-D scores was demonstrated in patients with major depressive disorder (MDD) at 15 and 28 days compared to placebo. Interim results for zuranolone in PPD and bipolar disorder (BPD) show promising reductions in HAM-D scores. Adverse effects included sedation, dizziness, and headache.

Place in Therapy: Allopregnanolone agonists seem to have a role in PPD when weighing the quick onset of action and potential risks of untreated PPD. The class of medications is limited by the single course for this indication and may fit as a bridge to maintenance therapy with selective serotonin reuptake inhibitors (SSRIs). Brexanolone, specifically, is hindered by the long infusion time, hospitalization associated with administration, and risk evaluation and mitigation strategy program. Zuranolone may also have a role in MDD or BPD, but more data are needed.

Conclusion: Allopregnanolone agonists present a novel mechanism of action in the treatment of depressive disorders. Clinical trials and interim results support significant reductions in depression scores for brexanolone in PPD, and for zuranolone in PPD, MDD, and BPD.

Keywords: postpartum, bipolar, brexanolone, zuranolone

\section{Introduction}

Mental illnesses are highly prevalent in the United States (USA), with approximately 51.5 million (20\%) of US adults indicating a mental illness in a 2019 survey. ${ }^{1}$ Major depressive disorder (MDD) affected nearly 17.3 million adults in the USA as of 2017, and between 2001 and 2003 there were $2.8 \%$ of US adults diagnosed with bipolar disorder (BPD). ${ }^{2,3}$ Effective treatment of depressive disorders is imperative as the cost is high. Approximately $15 \%$ of people with MDD will attempt and/or think about dying by suicide. ${ }^{4}$ Currently available therapies for the treatment of depression and adjunctive treatment of BPD primarily target
Correspondence: Lauren D Leader Michigan Medicine, Department of Pharmacy Services, I540 E. Medical Center Dr., Ann Arbor, MI, 48109 Tel + I 734 647-5938

Email leaderla@med.umich.edu 
modulation of the neurotransmitters serotonin and norepinephrine. These therapies are limited by delayed onset (46 weeks), lack of acute improvement in suicidality, and risk of mania when used to treat bipolar depression. ${ }^{5,6}$ Increasing evidence supports the idea that neuroactive steroids, specifically allopregnanolone, represent a novel target for the treatment of depression. ${ }^{7}$ In the presence of acute stress, allopregnanolone concentrations increase significantly. ${ }^{7}$ The increase in allopregnanolone is hypothesized to be neuroprotective. However, under chronic stress and in MDD, decreases in allopregnanolone concentrations in the central nervous system have been demonstrated, resulting in altered gamma-aminobutyric acid (GABA) and glutamate transmission. ${ }^{8}$ Allopregnanolone is thought to improve depressive symptoms through modulation of the $\mathrm{GABA}_{\mathrm{A}}$ pathway and regulation of the hypothalamic-pituitary-adrenocortical (HPA) axis, which facilitates recovery of physiological homeostasis. ${ }^{9}$ Allopregnanolone agonists are being developed as a potential treatment option for mental health disorders, exploiting the $\mathrm{GABA}_{\mathrm{A}}$ pathway activated by neuroactive steroids.

For decades, $\mathrm{GABA}_{\mathrm{A}}$ receptors have been considered to have a role in the pathophysiology of depression; however, the precise mechanisms are still not completely understood. The $\mathrm{GABA}_{\mathrm{A}}$ receptor complex is an ion channel comprised of five subunits with a multitude of binding sites. With 19 known subunits (six $\alpha$; three each $\beta, \gamma$, and $\rho$; and one each $\delta, \varepsilon, \pi$, and $\theta$ ), there are over a million different potential receptor conformations; however, fewer than 50 are thought to naturally occur and even fewer have pharmacological targets. In animals, decreases in $\alpha_{2^{-}}, \gamma_{2^{-}}$, and $\delta$-subunits have been associated with symptoms of depression and anxiety. ${ }^{10}$ In addition, decreased $\delta$-subunits have been associated with maternal neglect, and agonists of GABA receptors with the $\delta$-subunit (eg, allopreganolone) have been linked to improved maternal care. The benzodiazepines, for example, bind preferentially to receptors with $\alpha$ - and $\gamma$-subunits with a binding site between the two subunits. ${ }^{11}$ Throughout the 1980 s, numerous clinical trials evaluated the effect of benzodiazepines on depressive symptoms. ${ }^{12,13}$ Despite initial promising results, benzodiazepines were abandoned as primary treatment for depression owing to a lack of consistent benefit on core symptoms as well as a better understanding of the risks of abuse/dependence and possible association with worsening depression. ${ }^{14}$ Although benzodiazepines are still commonly used in practice for depression, current treatment guidelines relegate benzodiazepines to short-term adjunctive treatment of anxiety or catatonia associated with depressive episodes. ${ }^{5,15,16}$

The neurosteroid and metabolite of progesterone, allopregnanolone, also acts as a $\mathrm{GABA}_{\mathrm{A}}$ receptor modulator with preferential activity at receptors with a $\delta$-subunit. While benzodiazepines are thought to bind only to intrasynaptic sites, allopregnanolone also binds to extrasynaptic sites, which may have clinical implications (eg, differences in tolerance over time). ${ }^{17}$ The full implications of the extrasynaptic binding are not known at this time. The interplay among progesterone, allopregnanolone, and GABA receptors is complex and can vary throughout the menstrual cycle and pregnancy. ${ }^{18}$ In addition, evidence suggests that the conformation of GABA receptors themselves and the subunit make-up can change in different hormonal and mood states. ${ }^{18,19}$

Brexanolone, the first synthetic version of allopregnanolone, was approved in 2019 for the treatment of major depression after pregnancy, also known as postpartum depression (PPD). ${ }^{20}$ Allopregnanolone levels increase steadily throughout pregnancy and decrease drastically to normal concentrations in the postpartum period, indicating that allopregnanolone may provide a specific pathophysiology for PPD. ${ }^{21}$ Brexanolone is an intravenous infusion administered stepwise over 60 hours, making the coordination of administration challenging. In addition, brexanolone has a risk evaluation and mitigation strategy (REMS) program due to excessive sedation and loss of consciousness. $^{20,21}$ A heterocyclic analogue of allopregnanolone, zuranolone, has been investigated as an oral alternative for treatment of PPD and other psychological and neurological disorders. ${ }^{17}$ This paper aims to review the current efficacy and safety literature available for all forms of allopregnanolone agonists and to discuss the role of these medications in the treatment of mental health disorders.

\section{Literature Search}

A PubMed search of English-language articles was conducted, and clinical trials returned with the keywords of brexanolone (SAGE-547) and zuranolone (SAGE-217) were extracted. Since the aim of this review was to review clinical safety and efficacy, included articles were limited to phase II and phase III trials. To find zuranolone clinical trials in progress, the National Institutes of Health US National Library of Medicine Clinical Trials Database (clinicaltrials.gov) was utilized. After visiting the website 
of the drug manufacturer (Sage Therapeutics) to search for any preliminary results on zuranolone, a press release was found which referenced an abstract from the Psych Congress Annual Meeting. This led to a review of all 2019 and 2020 Psych Congress Annual Meeting abstracts and posters for trials involving zuranolone. Studies included in the review are summarized in Table 1.

\section{Results}

\section{Brexanolone: Clinical Efficacy and Safety}

Brexanolone has been studied for efficacy and safety for the treatment of PPD in one phase II (PPD-202A) and two phase III clinical trials (PPD-202B and PPD-202C). All three studies included patients who were aged 18-45 years, $\leq 6$ months postpartum, and had stopped lactating or were not actively breastfeeding. Patients with active psychosis, a medical history of seizures or BPD, or attempted suicide with the index case of PPD were excluded. Patients were diagnosed with a major depressive episode that began no earlier than the third trimester and no later than 4 weeks following delivery, and were required to have a 17-item Hamilton Rating Scale for Depression (HAM-D) total score of $\geq 26$ (for PPD-202A and 202B) or 20-25 (for PPD-202C), indicating moderate to severe depression. Patients were allowed to continue their current stable antidepressant regimen, but could not initiate any other pharmacotherapy regimen until the 60hour infusion and 12-hour post-infusion assessment had been completed. ${ }^{21,22}$ The primary outcome for all three studies was the change in baseline HAM-D total scores at the end of treatment.

\section{Study 202A}

Twenty-one patients were included in this phase II, double-blind, randomized, placebo-controlled trial. Patients were randomized $1: 1$ to a single 60 -hour infusion of either brexanolone $90 \mu \mathrm{g} / \mathrm{h}(\mathrm{n}=10)$ or placebo $(\mathrm{n}=11) .{ }^{22}$ Patients had a median age of 28.1 years (range 20-40) and a median body mass index (BMI) of $31 \mathrm{~kg} / \mathrm{m}^{2}$. Nearly two-thirds of patients were Black. Previous psychiatric disorder diagnoses, except for anxiety, and antidepressant use were similar between groups, with three patients in each group taking antidepressants at baseline. Baseline HAM-D scores were 28.1 and 28.8 in the brexanolone and placebo groups, respectively.

At the end of the 60-hour infusion, the mean reduction in HAM-D total score was 21.0 points in the brexanolone group and 8.8 points in the placebo group (least squares
[LS] mean difference -12.2 points, 95\% CI -20.77 to $-3.67, p=0.0075$; effect size $=1.2$ ). This effect was maintained during the follow-up period after the infusion up to 30 days (LS mean difference -11.9 points, $p=0.0095$ ). In addition, Montgomery-Asberg Depression Rating Scale (MADRS) scores were significantly different at 60 hours and 30 days (mean difference -12.1, $p=0.0104$; and $-11.19, p=0.0100$, respectively). Remission rates, defined as HAM-D total score $\leq 7$, were higher in the brexanolone group compared to the placebo group, with $70 \%$ of patients achieving remission at both 60 hours and 30 days compared to $9 \%$ and $18 \%$, respectively, in the placebo group.

Brexanolone was well tolerated, with no deaths, serious adverse events, or discontinuations occurring in either group. Adverse events were less common in the brexanolone group $(n=4)$ compared to the placebo group $(n=8)$. The most common adverse effects of brexanolone included dizziness $(20 \%)$ and somnolence $(20 \%)$. One patient in the placebo group had a severe treatment-emergent adverse event of insomnia.

\section{Study 202B}

In total, 138 patients were included in this phase III, double-blind, randomized, placebo-controlled trial. Patients were randomized to a single 60-hour infusion of either brexanolone $60 \mu \mathrm{g} / \mathrm{h}(\mathrm{BRX} 60, \mathrm{n}=47)$, or brexanolone $90 \mu \mathrm{g} / \mathrm{h} \quad(\mathrm{BRX} 90, \mathrm{n}=45)$, or placebo $(\mathrm{n}=46) .^{21}$ Additional exclusion criteria for studies 202B and 202C were women with a history of renal or hepatic failure, anemia, or known allergy to the study drug, or who were receiving electroconvulsive therapy. Baseline characteristics were similar between the three groups. Patients had a mean age of 27.8 years and a mean BMI of $30.7 \mathrm{~kg} / \mathrm{m}^{2}$. Nearly two-thirds of the patients were White. Treatment was discontinued in nine of 122 patients who received the study drug; one in the placebo group (lost to follow-up), three in the BRX60 group (two withdrew consent and one was lost to follow-up), and five in the BRX90 group (three withdrew consent and two were lost to follow-up). Baseline HAM-D scores were 29.1, 28.4, and 28.6 in the BRX60, BRX90, and placebo groups, respectively.

At the end of the 60-hour infusion, the mean reduction in HAM-D total score was 19.5 points in the BRX60 group, 17.7 points in the BRX90 group, and 14 points in the placebo group (LS mean difference -5.5 points, $95 \%$ CI -8.8 to $-2.2, p=0.0013$; and LS mean difference -3.7 points, $95 \% \mathrm{CI}-6.9$ to $-0.5, p=0.0252$, respectively). This 


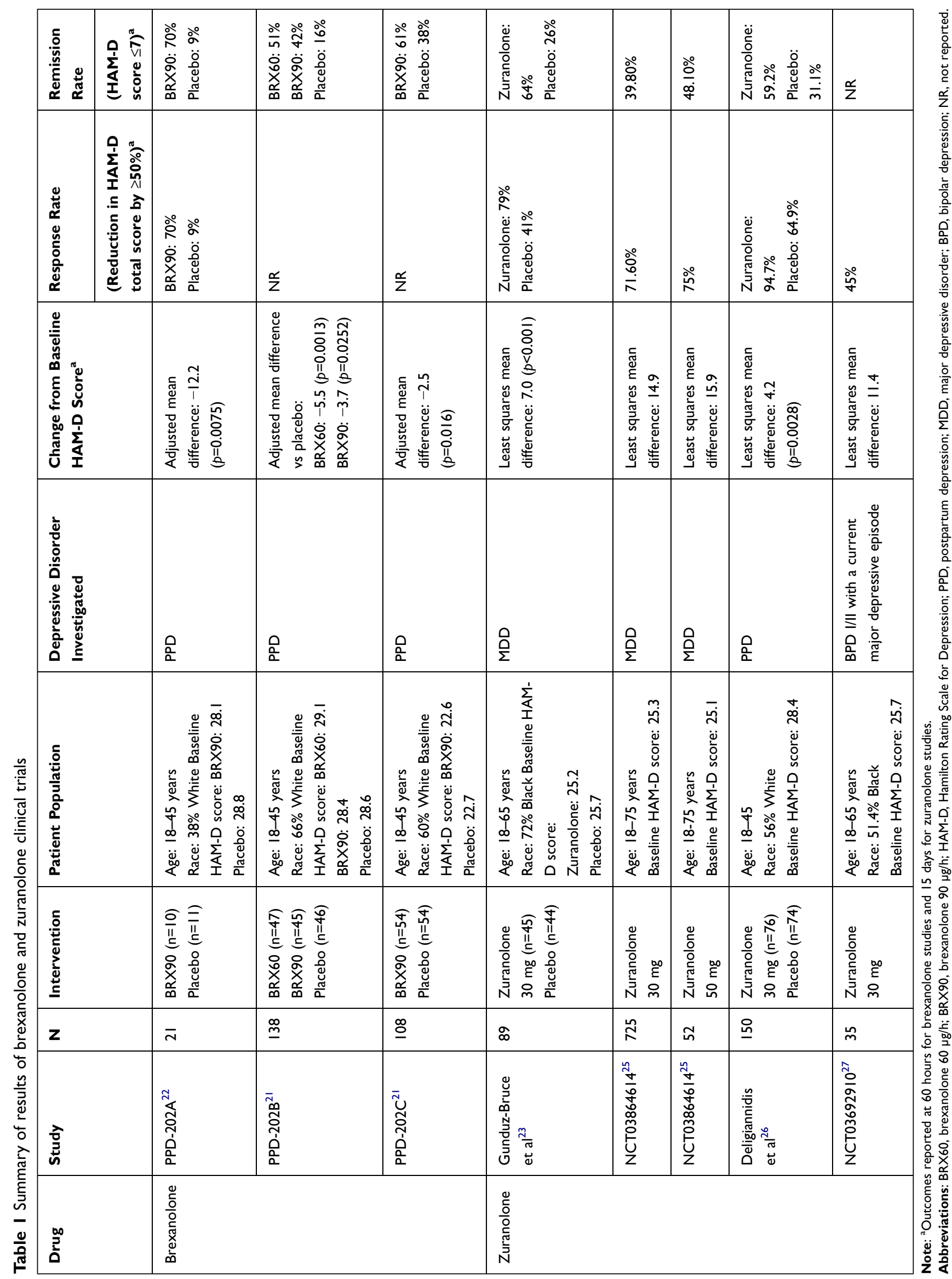


effect was maintained during the follow-up period after the infusion up to 30 days for both the BRX60 and BRX90 groups (LS mean difference -5.6 points, $95 \% \mathrm{CI}-9.5$ to $-1.8, p=0.0044$; and LS mean difference -3.8 points, $95 \%$ CI -7.6 to $0.0, p=0.0481$, respectively). Remission rates, defined as HAM-D total score $\leq 7$, were higher in the BRX60 group compared to placebo, with $51 \%$ of patients achieving remission at 60 hours, but not 30 days, compared to $16 \%$ in the placebo group. Patients in the BRX90 group did not have significantly higher remission rates compared to the placebo group at either 60 hours or 30 days.

Brexanolone was well tolerated, with no deaths, serious adverse events, or discontinuations occurring in either group. The total number of adverse events was similar across the three groups (BRX60 $=90, \mathrm{BRX} 90=22$, and placebo=22) The most common adverse effects of BRX60 included headache $(18 \%)$, somnolence $(18 \%)$, and dizziness $(16 \%)$. The most common adverse effects of BRX90 included headache (15\%), somnolence (5\%), and dizziness (15\%). One patient in the BRX60 group had two serious adverse events of suicidal ideation and an intentional overdose attempt, which were not considered to be related to the study drug. Another patient experienced somnolence and loss of consciousness during treatment, but completed treatment following interruption of the infusion with rapid resolution of adverse events.

\section{Study $202 C$}

A total of 108 patients were included in this phase III, double-blind, randomized, placebo-controlled trial. Patients were randomized to a single 60 -hour infusion of either BRX90 $(n=54)$ or placebo $(n=54)$. Patients had a mean age of 27.9 years and a mean BMI of $32.3 \mathrm{~kg} / \mathrm{m}^{2}{ }^{21}$ Sixty percent of the patients were White. Treatment was discontinued in four of 108 patients who received the study drug; one in the placebo group (lost to follow-up) and three in the brexanolone group (one withdrew consent, one adverse event, and one lost to follow-up). Baseline HAM-D scores were 22.6 and 22.7 in the BRX90 and placebo groups, respectively.

At the end of the 60-hour infusion, the mean reduction in HAM-D total score was 14.6 points in the BRX90 group and 12.1 points in the placebo group (LS mean difference -2.5 points, $95 \%$ CI -4.5 to $-0.5, p=0.0160$ ). This effect was not maintained during the follow-up period after the infusion up to 30 days (LS mean difference 0.5 points, $95 \% \mathrm{CI}-2.0$ to $3.1, p=0.6710$ ). Remission rates, defined as HAM-D total score $\leq 7$, were higher in the BRX90 group compared to placebo, with $61 \%$ of patients achieving remission at 60 hours, but not 30 days, compared to $38 \%$ in the placebo group.

Brexanolone was well tolerated, with no deaths, serious adverse events, or discontinuations occurring in either group. The total number of adverse events was similar $(B R X 60=90, B R X 90=9$, and placebo $=6$ ). The most common adverse effects of BRX90 included headache (18\%), infusion site pain $(10 \%)$, nausea $(10 \%)$, and dizziness (10\%). One patient in the BRX90 group had two serious adverse effects of altered state of consciousness and syncope, which were moderate in intensity and were related to treatment. Two patients in the BRX90 group had severe adverse events of fatigue $(n=1)$ and presyncope $(n=1)$. Across both studies 202B and 202C, five patients had excessive sedation which was determined to be due to brexanolone. In all cases, infusion was stopped and the events resolved within 90 minutes. All patients who lost consciousness regained consciousness within 15 minutes of infusion cessation. There were no differences in laboratory parameters, vital signs, or electrocardiograms between brexanolone and placebo groups across both studies.

These studies demonstrated the efficacy and safety of brexanolone for PPD, with statistically and clinically meaningful reductions in HAM-D scores compared to placebo. The antidepressant effect was rapid and the majority of patients achieved remission (51-70\%) at the end of the 60-hour infusion. Although brexanolone was not consistently superior to placebo at 30 days in all three studies, the antidepressant effect was sustained in patients with both moderate and severe PPD, and no patients relapsed after treatment. These studies are limited by the short follow-up period of 30 days and significant placebo response rate, although that is common for antidepressant studies.

\section{Zuranolone: Clinical Efficacy and Safety}

Zuranolone efficacy and safety data have been published in one phase II study. In this double-blind trial, 89 patients were randomized 1:1 to receive either zuranolone $30 \mathrm{mg}$ $(n=45)$ or placebo $(n=44)$ daily for 14 days. ${ }^{23}$ Patients aged 18-65 years who had a diagnosis of MDD with a HAM-D score of 22 or higher were included in this trial. Patients could continue stable doses of antidepressants if they had been prescribed for at least 30 days. Initiation of new antidepressants was not allowed during the 14-day treatment period, but antidepressants could be 
adjusted or added during the 4 weeks after the treatment period, at which time secondary outcomes were assessed. Patients were excluded if they had a history of a suicide attempt or treatment-resistant depression, a recent history or current clinically significant manifestations of other acute or chronic medical conditions, a positive pregnancy test, a history of seizures, or a history of BPD, schizophrenia, or schizoaffective disorder. The primary outcome was change in 17-item HAM-D total scores from baseline at 15 days.

Patients had a mean age of 43.7 years and a mean BMI of $29.95 \mathrm{~kg} / \mathrm{m}^{2}$, and $72 \%$ were Black. The use of antidepressants at baseline was similar between the groups, with 12 patients in the zuranolone group and 10 patients in the placebo group using antidepressants. Baseline HAM-D scores were 25.2 and 25.7 in the zuranolone and placebo groups, respectively. On day 15 , the mean reduction in HAM-D score was 17.4 in the zuranolone group and 10.3 in the placebo group (LS mean difference $-7.0,95 \% \mathrm{CI}-10.2$ to $-3.9, p<0.001)$. This effect was maintained during the follow-up period up to 28 days (LS mean difference $-4.1,95 \% \mathrm{CI}-7.6$ to -0.5 ), but not for days 35 and 42 (LS mean difference -2.3 , $95 \%$ CI -6.0 to 1.4 for both). In addition, MADRS scores were significantly different at 15 days (LS mean difference $-7.6,95 \% \mathrm{CI}-12.3$ to -2.8 ), but not at 28 , 35 , or 42 days. The response rate at 15 days, defined as a reduction of at least 50\% from baseline HAM-D scores, was $79 \%$ in the zuranolone group and $41 \%$ in the placebo group (OR 9.6, 95\% CI 2.9 to 31.6). Remission rates, defined as HAM-D score $\leq 7$, were higher in the zuranolone group (64\%) compared to the placebo group (26\%) (OR 5.3, 95\% CI 2.1 to 13.3).

Zuranolone was relatively well tolerated, with no deaths, serious adverse events, or discontinuations occurring in either group. Adverse events, none of which was serious or severe, were more common in the zuranolone group $(n=24)$ than in the placebo group $(n=20)$. The most common adverse effects of zuranolone were headache (18\%), dizziness (11\%), and nausea (11\%). Six patients in the zuranolone group had dose reductions from $30 \mathrm{mg}$ to $20 \mathrm{mg}$, five for adverse events: dizziness $(\mathrm{n}=2)$, somnolence $(n=1)$, sedation $(n=1)$, nausea/vomiting $(n=1)$, and sleepiness $(n=1)$.

In the first phase III clinical trial, 581 patients with MDD were randomized to zuranolone $20 \mathrm{mg}, 30 \mathrm{mg}$, or placebo daily for 14 days. $^{24}$ There was minimal separation from placebo at day 15 (LS mean difference -1.4, $p=0.115)$ in the $30 \mathrm{mg}$ group and no separation in the $20 \mathrm{mg}$ group. At day 42, the reduction in depressive symptoms was maintained; however, the difference between zuranolone and placebo was not significant (LS mean difference $-0.5, p=0.807$ ). A post-hoc analysis was performed to evaluate the effects of performance factors on the primary outcome at day 15. Patients with measurable serum concentrations of zuranolone, those with HAM-D >24, and those with both measurable drug serum concentrations and HAM-D >24 had significant differences from placebo. Adverse effects in this trial are similar to those reported in other zuranolone trials discussed in this review. The investigators of this study concluded that the $9 \%$ of patients with undetectable drug serum concentrations may have significantly contributed to the negative findings.

\section{Interim Reports: MDD}

An interim report of a phase III, open-label, one-year longitudinal study (NCT03864614) presented at Psych Congress 2020 assessed a 14-day course of $30 \mathrm{mg}$ daily of zuranolone in patients aged 18-75 years with a diagnosis of MDD (HAM-D $\geq 20$ ). ${ }^{25}$ Patients were included if they had a diagnosis of MDD with symptoms that had been present for at least 4 weeks and a MADRS total score of $\geq 28$ and HAM-D total score of $\geq 20$. Patients were excluded if they had a suicide attempt associated with the index MDD episode, had a history of BPD, schizophrenia, or schizoaffective disorder, or had undergone treatment with vagal nerve stimulation or electroconvulsive therapy, or used any form of ketamine during the index MDD episode. ${ }^{25}$

The primary outcome was change in HAM-D scores from baseline at 15 days. In total, 725 patients, with a mean baseline HAM-D score of 25.3, were treated with $30 \mathrm{mg}$ of zuranolone. At day 15 of the initial course of therapy, the mean reduction in HAM-D scores was -14.9, the response rate ( $\geq 50 \%$ reduction in HAM-D scores) was $71.6 \%$, and the remission rate (HAMD $\leq 7$ ) was $39.8 \%$. The most commonly reported adverse events were somnolence $(9.5 \%)$, headache $(8.7 \%)$, and dizziness $(5.4 \%)$, of mild or moderate severity.

Patients who responded to therapy in the first phase $(n=494)$ could be retreated with a course of zuranolone (30 mg until May 2020, and then $50 \mathrm{mg}$ ). The majority of patients $(44.5 \%)$ required only the initial zuranolone course, while $26.7 \%$ used two total courses, $13.4 \%$ used three total courses, $10.3 \%$ used four total courses, and 
$5.5 \%$ used a total of five courses. In the interim sample of patients who were initially treated with $30 \mathrm{mg}$ who then received repeat treatment with $50 \mathrm{mg}$, patients had higher rates and intensity of adverse events. Patients were reassessed for the need for repeat treatment every 14 days and were given another course if HAM-D scores were $\geq 20$ or Patient Health Questionnaire-9 scores $\geq 10$. There was a minimum of 56 days between zuranolone courses.

A new cohort initially treated with $50 \mathrm{mg}$ of zuranolone $(n=52)$ had a mean HAM-D score of 25.1 at baseline. There was a change in HAM-D scores from baseline of $15.9 ; 75 \%$ of patients responded to treatment and $48.1 \%$ achieved remission. Of the 76 patients with safety data available from the $50 \mathrm{mg}$ cohort, adverse events were similar to the $30 \mathrm{mg}$ cohort. The most frequently reported adverse events were somnolence, dizziness, sedation, headache, and tremor of mild or moderate severity. .

A phase III, multicenter, double-blind, placebo-controlled study of zuranolone in MDD is completed, but not results are not yet available (NCT04442490). In addition, a phase III, randomized, double-blind study comparing zuranolone plus an antidepressant to placebo is recruiting, (NCT04476030).

\section{Interim Analysis: PPD}

An interim report of a phase III, double-blind, parallelgroup, placebo-controlled trial (NCT02978326) presented at the American Society of Clinical Psychopharmacology 2019 Meeting assessed a 14-day course of zuranolone $30 \mathrm{mg}$ daily $(\mathrm{n}=76)$ or placebo $(\mathrm{n}=74)$ once daily. ${ }^{26}$ Inclusion criteria for this trial were the same as those for the brexanolone studies 202A and 202B. Patients had a mean age of 28.4 years, $56 \%$ were White, and the baseline HAM-D score was 28.4 in the zuranolone group and 28.8 in the placebo group.

The primary outcome was change in HAM-D scores from baseline at 15 days. At day 15, the mean reduction in HAM-D scores was 17.8 in the zuranolone group and 13.6 in the placebo group $(p=0.0028)$. The response rate, defined as $\geq 50 \%$ reduction in HAM-D scores, was greater in the zuranolone group compared to the placebo group (94.7\% vs $64.9 \%, p=0.0049$ ), as was the remission rate, defined as HAM-D total score $\leq 7 \quad(59.2 \%$ vs 31.1 , $p=0.0110$ ). The most common adverse effects were somnolence $(15 \%)$, headache $(9 \%)$, dizziness $(8 \%)$, upper respiratory tract infection $(8 \%)$, and diarrhea $(6 \%)$, most of which were mild or moderate. Six patients experienced severe adverse events, three from the zuranolone group (one sedation, one confusion, one migraine) and three from the placebo group (one back pain and muscle spasms, one headache and oropharyngeal pain, and one menorrhagia). Two patients, one from each group, experienced serious adverse effects. This study has completed recruitment. Another phase III, double-blind, placebo-controlled study (NCT04442503) evaluating the efficacy and safety of zuranolone for PPD is underway, with an estimated completion date of December 2021.

\section{Interim Analysis: BPD}

An interim analysis of an open-label, phase II trial (NCT03692910) presented at Psych Congress 2020 assessed a 14-day course of $30 \mathrm{mg}$ of zuranolone in patients aged 18-65 years with a documented history of mania/hypomania and a diagnosis of BPD I or BPD II, in a current major depressive episode (HAM-D $\geq 22$ ). ${ }^{27}$ Patients with a history of rapid cycling BPD or a depressive disorder with mixed features, or history of a suicide attempt or current suicidal ideation with plan, were excluded. Primary outcome measures were the safety and tolerability of zuranolone as assessed by the frequency and severity of adverse events, Columbia Suicide Severity Rating Scale, and Young Mania Rating Scale. Secondary outcome measures were the change in HAM-D and MADRS total scores from baseline at 15 days.

Thirty-five patients with a mean age of 47.6 years were included in part one of this clinical study. The majority of patients had BPD I (88.6\%), and were Black (51.4\%), and female $(65.7 \%)$, with a mean BMI of $32.3 \mathrm{~kg} / \mathrm{m}^{2}$ and a mean baseline HAM-D total score of 25.7. Zuranolone was generally well tolerated in this population, with no severe or serious adverse events, no mania, no required dose reductions due to adverse events, and no increased suicidal ideation or behavior. Two patients experienced mild hypomania, which occurred in the treatment followup period. The most common adverse effects reported were somnolence $(11.4 \%)$ and headache $(8.6 \%)$. With regard to efficacy outcomes, there was a reduction in MADRS total score $(-15.5)$ and a reduction in HAM-D total score (-11.4), both sustained through day 42 . Response, defined as $\geq 50 \%$ reduction of HAM-D total score, was achieved in $45 \%$ at day 15 , with $50 \%$ achieving a response at day 42 . This study is phase A of a two-part study, with plans for a double-blind, randomized, placebocontrolled, parallel group trial of zuranolone compared to placebo in BPD from Sage Therapeutics. 
These studies have demonstrated the efficacy and safety of zuranolone for a variety of depressive illnesses for patients with severe depression, including PPD, MDD, and BPD. Antidepressant effects appear to be rapid and have a sustained effect, with high response rates (45-94.7\%) and remission rates $(39.8-64 \%)$ at the end of the 14-day treatment course, in some cases up to 42 days. These studies are limited by the lack of complete data, short follow-up periods, and significant placebo response rates.

\section{Discussion}

\section{Place in Therapy (PPD)}

PPD is a common complication of pregnancy, as one in eight women reports symptoms of depression after giving birth. ${ }^{28}$ Perinatal depression includes major and minor depressive episodes that begin during pregnancy or in the first 12 months after delivery. ${ }^{29}$ As allopregnanolone agonists have not been studied during pregnancy, these medications should be limited for use in moderate to severe PPD after delivery has occurred.

Exogenous allopregnanolone (brexanolone) and the analogue zuranolone represent first-in-class medications with regard to mechanism and indication for PPD. Allopregnanolone levels peak during the third trimester and decline rapidly following childbirth. While their unique mechanism of action is not fully understood, the hypothesis is that administering allopregnanolone agonists could restore allopregnanolone concentrations and reset neural networks, resulting in improved PPD symptoms. ${ }^{20}$

In clinical trials, brexanolone is unique in the treatment of depression in that response and remission occur rapidly, within 60 hours, with a sustained effect to at least 30 days. In addition, the response and remission rates in trials exceed those seen with traditional antidepressants $(\sim 50 \%$ and $\sim 30 \%$, respectively). Mechanistically, if PPD is due to a rapid decline in allopregnanolone levels, exogenous administration over days to allow a smoother decline in levels makes clinical sense. This relatively rapid time to improvement in depressive symptoms (60 hours for brexanolone, 15 days for zuranolone) provides a potential advantage over other antidepressants such as selective serotonin reuptake inhibitors (SSRIs), which can take 46 weeks to provide remission. It should be noted, however, that brexanolone and zuranolone have only been studied in comparison to placebo at this time. In addition, these medications are only given once for their allotted treatment time (60 hours for brexanolone, 14 days for zuranolone) and not continued for months or years, therefore reducing the potential pill burden.

It should be noted that in clinical trials of brexanolone, the rate of historical non-PPD depression ranged from $24 \%$ to $60 \%$ and the rate of previous PPD ranged from $27 \%$ to $70 \%$. Based on the available published data, it is not clear whether a past history of MDD impacts the likelihood of response or remission. Future studies should focus on differentiating new-onset or recurrent PPD from recurrence of MDD in the postpartum period. Defining the optimal time for administration (eg, the point at which allopregnanolone levels stabilize postpartum) and whether there is a role for prophylactic dosing in women with a history of PPD are additional questions to be answered.

The 60-hour continuous infusion administration of brexanolone has several barriers that should be considered by both clinicians and patients. The REMS program requires the infusion to be administered in a medically supervised setting where patients can be continuously monitored for excessive sedation and loss of consciousness. Patients receiving brexanolone must not be the primary caregiver of their children during the infusion. ${ }^{30}$ Furthermore, the cost of the medication $(>\$ 30,000$ per 60-hour infusion) and the cost of admission to a medically supervised setting could limit access for some patients. Healthcare settings should utilize a multidisciplinary approach to develop optimal procedures for REMS program compliance, patient safety, and cost reimbursement.

\section{Place in Therapy (MDD and BPD)}

The trial of zuranolone for MDD did not demonstrate the same maintenance of response after the end of treatment, with improvement dissipating by day 28 (14 days after the end of treatment). Furthermore, in the open-label study, over $50 \%$ of patients required additional courses of treatment. The BPD study, however, did find a sustained improvement as far as day 42 , with a slight improvement over the initial response rate. This may be related to the plasticity of $\mathrm{GABA}_{\mathrm{A}}$ receptors and differential effects of allopregnanolone in MDD, BPD, and PPD. The rapid improvement in MDD symptoms over 14 days without sustained benefit harks back to the clinical trials of benzodiazepines for depression. Future studies should include concurrent initiation of traditional antidepressants as maintenance. With two patients experiencing hypomania in the follow-up period, the risk of mood switch in BPD and the impact of concurrent mood stabilizers also warrant assessment. 
Despite rapid improvement in symptoms, patients with treatment-resistant depression or suicidality were excluded from all of the clinical trials discussed. While this is standard in clinical trials of antidepressants, it would be beneficial to include these patients in future clinical trials as they are often the most in need of rapid response. In addition, given the widespread use of benzodiazepines in patients with depression, the clinical impact of concurrent use of two different $\mathrm{GABA}_{\mathrm{A}}$ receptor modulators requires more understanding.

\section{Conclusion}

The novel mechanism of allopregnanolone agonists on $\mathrm{GABA}_{\mathrm{A}}$ receptors provides a promising innovation in the treatment of depressive disorders. The quick onset of remission with these agents is a benefit over standard therapies and they may have a niche as an induction agent or a bridge to maintenance therapy with SSRIs. Considering the role of endogenous allopregnanolone in PPD, the benefit of these agents may outweigh the costs and severe risks of untreated PPD. In contrast, in MDD, the mechanism of allopregnanolone agonists mirrors that of benzodiazepines, which are no longer recommended, potentially indicating a lack of benefit in this indication. The data, overall, are encouraging, but the results of further studies with direct comparisons and combination drugs are eagerly anticipated.

\section{Disclosure}

Dr. Leader reports consulting for Wolters-Kluwer on medication use in pregnancy and lactation and Pfizer, Inc. on their menopause portfolio. Dr. Cooke reports participation in a Sage Therapeutics, Inc. Site Activation Advisory Board Meeting in November 2019. The authors report no other conflicts of interest in this work.

\section{References}

1. Center for Behavioral Health Statistics and Quality. 2019 National Survey on Drug Use and Health: Methodological Summary and Definitions. Rockville, MD: Substance Abuse and Mental Health Services Administration; 2020.

2. Center for Behavioral Health Statistics and Quality. 2017 National Survey on Drug Use and Health: Methodological Summary and Definitions. Rockville, MD: Substance Abuse and Mental Health Services Administration; 2018.

3. National Comorbidity Survey (NSC). Harvard Medical School; 2007. Available from: https://www.hcp.med.harvard.edu/ncs/ index.php.DataTable 2:12-monthprevalenceDSM-IV/WMHCIDIdisordersbysexandcohort. Accessed March 27, 2021.
4. Orsolini L, Latini R, Pompili M, et al. Understanding the complex of suicide in depression: from research to clinics. Psychiatry Investig. 2020;17(3):207-221. doi:10.30773/pi.2019.0171

5. Major Depressive Disorder Work Group. Practice Guideline for the Treatment of Patients with Major Depressive Disorder. 3rd ed, Fochtmann LJ. ed: American Psychiatric Association; 2010.

6. Gitlin MJ. Antidepressants in bipolar depression: an enduring controversy. Int $J$ Bipolar Disord. 2018;6(1):25. doi:10.1186/ s40345-018-0133-9

7. Bali A, Singh Jaggi A. Multifunctional aspects of allopregnanolone in stress and related disorders. Prog Neuropsychopharmacol Biol Psychiatry. 2014;48:64-78. doi:10.1016/j.pnpbp.2013.09.005

8. Lüscher B, Möhler H. Brexanolone, a neurosteroid antidepressant, vindicates the GABAergic deficit hypothesis of depression and may foster resilience. F1000Res. 2019;8:1-13. doi:10.12688/ f1000research.18758.1

9. Schule C, Nothdurfter C, Rupprecht R. The role of allopregnanolone in depression and anxiety. Prog Neurobiol. 2014;113:79-87. doi:10.1016/j.pneurobio.2013.09.003

10. Engin E, Benham RS, Rudolf U. An emerging circuit pharmacology of $\mathrm{GABA}_{\mathrm{A}}$ receptors. Trends Pharmacol Sci. 2018;39(8):710-732.

11. Stephens DN, King SL, Lambert JJ, et al. GABAA receptor subtype involvement in addictive behaviour. Genes Brain and Behav. 2017;16 (1):149-184

12. Birkenhäger TK, Moleman P, Nolen WA. Benzodiazepines for depression? A review of the literature. Int Clin Psychopharmacol. 1995;10:181-195. doi:10.1097/00004850-199510030-00008

13. Petty F, Trivedi MH, Fulton M, Rush JA. Benzodiazepines as antidepressants: does GABA play a role in depression? Biol Psychiatry. 1995;38(9):578-591. doi:10.1016/0006-3223(95)00049-7

14. Lim B, Sproule BA, Zahra Z, et al. Understanding the effects of chronic benzodiazepine use in depression: a focus on neuropharmacology. Int Clin Psychopharmacol. 2020;35 (5):243-253. doi:10.1097/YIC.0000000000000316

15. Soric MM, Paxos C, Dugan SE, et al. Prevalence and predictors of benzodiazepine monotherapy in patients with depression: a national cross-sectional study. J Clin Psych. 2019;80(4):18m12588.

16. Kennedy SH, Lam RW, McIntyre RS, et al. Canadian network for mood and anxiety treatments (CANMAT) 2016 clinical guidelines for the management of adults with major depressive disorder: section 3 . Pharmacological treatments. Can J Psychiatry. 2016;61(9):540-560. doi: $10.1177 / 0706743716659417$

17. Botella GM, Salituro FG, Harrison BL, et al. Neuroactive steroids. 2. $3 \alpha$-hydroxy-3 $\beta$-methyl-21(4-cyano-1 $H$-pyrazol-1'-yl)-19-nor-5 $\beta$ pregnan-20-one (SAGE-217): a clinical next generation neuroactive steroid positive allosteric modulator of the $(\gamma \text {-aminobutyric acid })_{\mathrm{A}}$ receptor. $J$ Med Chem. 2017;60:7810-7819. doi:10.1021/acs. jmedchem.7b00846

18. Bixo M, Ekberg K, Poromaa IS, et al. Treatment of premenstrual dysphoric disorder with $\mathrm{GABA}_{\mathrm{A}}$ receptor modulating steroid antagonist Sepranolone (UC1010) - a randomized controlled trial. Psychoneuroendocrinology. 2017;80:46-55. doi:10.1016/j. psyneuen.2017.02.031

19. Engin E, Benham RS, Rudolph U. An emerging circuit pharmacology of $\mathrm{GABA}_{\mathrm{A}}$ receptors. Trends Pharmacol Sci. 2018;39(8):710-732. doi:10.1016/j.tips.2018.04.003

20. Sage Therapeutics, Inc. Zulresso ${ }^{T M}$ (Brexanolone Infusion) [Package Insert]. Cambridge, MA: Sage Therapeutics, Inc; 2019.

21. Meltzer-Brody S, Colquhoun H, Riesenberg R, et al. Brexanolone injection in post-partum depression: two multicentre, double-blind, randomised, placebo-controlled, Phase 3 trials. Lancet. 2018;392 (10152):1058-1070. doi:10.1016/S0140-6736(18)31551-4

22. Kanes S, Colquhoun H, Gunduz-Bruce H, et al. Brexanolone (SAGE-547 injection) in post-partum depression: a randomised controlled trial. Lancet. 2017;390(10093):480-489. doi:10.1016/S01406736(17)31264-3. 
23. Gunduz-Bruce H, Silber C, Kaul I. Trial of SAGE-217 in Patients with Major Depressive Disorder. $N$ Engl J Med. 2019;381 (10):903-911. doi:10.1056/NEJMoa1815981.

24. Sage Therapeutics Reports Topline Results from Pivotal Phase 3 MOUNTAIN Study of SAGE-217 in Major Depressive Disorder [Internet]. Cambridge: Businesswire; 2019. Available from: https://www. businesswire.com/news/home/20191205005375/en/Sage-TherapeuticsReports-Topline-Results-Pivotal-Phase. Accessed May 17, 2021.

25. Sage Therapeutics Announces Positive Interim, Topline Zuranolone Safety and Tolerability Data from Open-Label SHORELINE Study in Patients with MDD [Internet]. Cambridge: sage Therapeutics; 2021. Available from: https://investor.sagerx.com/news-releases/newsrelease-details/sage-therapeutics-announces-positive-interim-toplinezuranolone. Accessed March 6, 2021.

26. Deligiannidis KM, Lasser R, Gunduz-Bruce H, et al. The ROBIN trial, a phase 3, double-blind, placebo controlled trial of zuranolone in postpartum depression. Poster presented at: psych Congress 2020 Virtual Experience. Virtual. 2020.
27. Gunduz-Bruce H, Lasser R, Nandy I, et al. Open-label, Phase 2 trial of the oral neuroactive steroid $\mathrm{GABA}_{\mathrm{A}}$ receptor positive allosteric modulator zuranolone in bipolar disorder I and II. Poster presented at: psych Congress 2020 Virtual Experience. Virtual. 2020.

28. Bauman BL, Ko JY, Cox S, et al. Vital signs: postpartum depressive symptoms and provider discussions about perinatal depression United States, 2018. MMWR Morb Mortal Wkly Rep. 2020;69:575-581. doi:10.15585/mmwr.mm6919a2

29. Gavin NI, Gaynes BN, Lohr KN, Meltzer-Brody S, Gartlehner G, Swinson T. Perinatal depression: a systematic review of prevalence and incidence. Obstet Gynecol. 2005;106(5, Part 1):1071-1083. doi:10.1097/01.AOG.0000183597.31630.db

30. Zulresso REMS Training for Healthcare Settings [internet]. Sage Therapeutics, Inc.; 2020. Available from: https://www.zulressorems. com/\#Public/Resources. Accessed March 1, 2021.

\section{Publish your work in this journal}

Drug Design, Development and Therapy is an international, peerreviewed open-access journal that spans the spectrum of drug design and development through to clinical applications. Clinical outcomes, patient safety, and programs for the development and effective, safe, and sustained use of medicines are a feature of the journal, which has also been accepted for indexing on PubMed Central. The manuscript management system is completely online and includes a very quick and fair peer-review system, which is all easy to use. Visit http://www. dovepress.com/testimonials.php to read real quotes from published authors. 